\title{
Reaction Time and Spatiotemporal Variables as Markers of Sprint Start Performance
}

\author{
Ioannis Kesoglou, Athanasia Smirniotou* \\ School of Physical Education and Sport Science, National and Kapodistrian University of Athens, Athens, Greece
}

\author{
Email address: \\ asmirn@phed.uoa.gr (A. Smirniotou) \\ ${ }^{*}$ Corresponding author
}

\section{To cite this article:}

Ioannis Kesoglou, Athanasia Smirniotou. Reaction Time and Spatiotemporal Variables as Markers of Sprint Start Performance. American Journal of Sports Science. Vol. 7, No. 3, 2019, pp. 121-126. doi: 10.11648/j.ajss.20190703.16

Received: July 29; Accepted: August 19, 2019; Published: September 3, 2019

\begin{abstract}
The purpose of the present study was to examine both the within-day and between-day reliability of sprint start variables. The reaction time and the premotor time (PMT) were used as markers of the reactive function. Additionally, 3-m time, first-step flight time, first-step length and first-step velocity were utilized as spatiotemporal markers of the sprint start. Prior to the reliability study, an extensive number of sprint start trials were performed to familiarize the participants. A repeated measures analysis of variance, intra-class correlation coefficient (ICC) and Cronbach's alpha reliability coefficient were used to assess the within-day and between-day reliability. The coefficient of variation (CV\%) was evaluated to determine the withinparticipant variation. Both the within-day and between-day spatiotemporal markers showed higher reliability with lower variability from the reactive function markers. This study shows that well-familiarized participants can perform a reliable sprint start performance both within a day and between days. We conclude that the reactive markers have a high variability within a day and between days and require further research to determine their dynamic role in the sprint start action. Therefore, spatiotemporal markers provide a more valid method of sprint start assessment.
\end{abstract}

Keywords: Familiarization, Premotor Time, Electromyography, Sprinting, Reliability

\section{Introduction}

Sprint starting action has been identified as an important factor in athletics by coaches and researchers. Kinematic, kinetic and electromyographic (EMG) variables have been investigated to assess the decisive role of the sprint start on sprinting performance [1]. Kinematic and kinetic (dynamic) refers to the mechanics that describe the motion analysis of the body joint moments and the body segments and the forces that cause the movement. Additionally, EMG analysis assesses the action potential of muscle contraction and the recruitment of muscle fibers [2].

Optimal starting performance requires advanced physiological and psychological abilities and the potential to produce horizontal forces [1] via a high level of motor coordination between the upper and lower body segments [3]. In the "set" position, a sprinter engages mental readiness from the starting gun to whole body maximal activation under specific physical and technical considerations such as the interblock distance, block obliquity, joint alignment and total body center of gravity $[1,4,5]$. The time interval between the gun signal and initiation of a response on the starting blocks is defined as reaction time (RT), and an athlete who performs more quickly than $100 \mathrm{~ms}$ is considered to have committed a false start. However, an athlete with adequate neuromuscular function could have a faster RT $(<100 \mathrm{~ms})$ [6]. The RT is an index of motor readiness (mental) and speed of the response (neural) initiation factor and encompasses premotor time (PMT) and motor time (MT). PMT refers to the time between the gun signal and the onset of the muscle action potential (electromyographic activity). The MT is the time interval between the onset of the 'muscle firing' and the force production that is generated by the feet against the block [2]. The RT performance is associated with individual factors such as age, the arousalattention state, practice-fatigue effects and motivational influences. RT is also associated with procedural factors, which are related to the nature of the stimuli and the response (simple RT and choice RT) with respect to the environmental conditions [7]. However, the sprint start is primarily a 
complex motor function that incorporates a simple RT [8] with the precise nature of the stimuli and the response known in advance, which can be preprogrammed via training. After the gun signal, a sequence of muscle activity occurs. A forward propulsion of the body is determined by the involvement of the leg muscles against the starting blocks and powerful arm movements [9]. The athlete starts moving from the acyclic to the cyclic phase of the starting action when the rear leg comes in contact with the ground. In light of reliability and applied research, many researchers have been interested in starting performance, analyzing athletic competitions and using experienced athletes under investigation $[2,3,10]$. However, it is hard to correlate the sprint start performance with the final distance time due to race tactics and the lack of evidence that relates the best personal distance time with the best starting performance. Thus, reliable measurements should be performed to investigate brief high-intensity actions, such as starting, and differentiate from the final distance time to allow the exact synoptic inference of the applied research [11-13].

The importance of the starting action in the total sprint performance was highlighted by Mero and Komi (1990) who observed a significant positive correlation between the MT and maximum horizontal forces on the blocks despite not finding a significant correlation between the RT and block velocity. In support of these findings, Smirniotou et al. (2008) reported no relationship between the RT and sprint performance, but the RT was well correlated with the vertical jump index (CMJ-SJ). Tønnessen et al. (2013) analyzed the IAAF's $100-\mathrm{m}$ data from the world championships (2003 to 2009) using different gender and age groups and concluded that RT ability is positively related to the final time of the running distance. It was also reported that the competitive level of the athletes and the entry standards set by the IAAF contributed to the results being representative. Recently, a biomechanical analysis of a wide stance compared to a normal stance on the block start showed that the width of the starting blocks affect the motion and the induced-force of the rear leg on the block, but no significant difference was found considering the entire block start performance. Additionally, the RT indicates a lack of absolute agreement and higher reliability [13]. In applied research, reliable RT measurements have to be obtained across a number of trials, which develops an attentional or response 'set' and ensures that the values are not unduly affected by an entire timescale that may occur in some trials [15]. To ensure that the participants understand the task instructions and are familiarized with the requirements of the task, it is also customary to provide some practice trials before the actual measurement of the RTs. To our knowledge, there are no studies that have explored the reliability of sprint start variables across a timescale using a starting device. Therefore, the aim of the present study was to examine the reliability of sprint start variables both within a day and between days to investigate the potential time-of-day effect. The RT and PMT were used as markers of the reactive function. Additionally, 3-m time, first-step flight time, first- step length and first-step velocity were used as spatiotemporal markers of the sprint start.

The following two hypotheses were formulated based on the literature review: 1 . The within-day reliability would be higher than the between-day reliability; and 2. Reactive function markers would be less reliable than spatiotemporal markers.

\section{Methods}

\subsection{Participants}

Eight healthy male students (mean \pm SD: age $19.63 \pm 0.9$ years; body mass $75.56 \pm 7.37 \mathrm{~kg}$; height $1.80 \pm 0.07 \mathrm{~m}$ ) who had limited experience in sprint start performance, were recruited from the university to take part in this study. All individuals had no history of disease or muscle-skeletal abnormalities and gave their written informed consent to participate. All procedures were fully explained, and the research protocol was approved by the university ethics board. Participants maintained a normal diet and were asked to refrain from vigorous exercise, alcohol and caffeine consumption during the study.

\subsection{Familiarization}

Prior to the reliability study, an extensive number of sprint start trials were performed to familiarize the participants. In the initial session, participants were tested to determine the most comfortable crouch position on the starting blocks. The feet were placed onto the blocks without the toes contacting the ground. The length of the rear and the front blocks were measured using a measurement tape (rear, $0.85 \mathrm{~m} \pm 0.06 \mathrm{~m}$; front, $0.63 \mathrm{~m} \pm 0.07 \mathrm{~m}$ ), and the inclinations of the blocks were set at $\approx 50^{\circ}$ on the front foot and $\approx 65^{\circ}$ on the rear foot [16]. The single-leg jump tests (countermovement and squat) were performed to determine the dominant leg (or the strongest leg) in relation to the block leg placement; further analysis showed no relationship between the jump tests and the participants' preferred block leg placement. Both the length and inclination of the blocks remained the same until the end of the study. In the following familiarization sessions, the participants performed five sprint start trials over a distance of ten meters in each session with five minutes of recovery between the trials. A specific warm-up protocol was applied prior to every session [17]. A photocell gate was placed at a distance of three meters and was connected to the starting device (Polifemo Radio LightMicrogate, Italy). After each trial, the RT (ReacTime-Lynx System Developers, Inc.), and the 3-m time was used as an acute feedback of the sprint start performance, and the average scores of the RT and 3-m time of the five trials was used for further analysis. The 3-m time was chosen as a block clearance-related time to provide results due to both its simplicity and the speed of the response. Subsequent familiarization sessions were performed every 24 to 30 hours until the participant's reaction time and three-meter time demonstrated repeatable results (plateau). We set the five 
sessions as the minimum number of sessions required for familiarization. Each participant performed five sprint starts in each session. The average score of the five trials was used for further analysis. The plateau effect was achieved within three to five sessions.

\subsection{Experimental Design}

A repeated measures design was achieved two to three days after the familiarization procedure. Participants performed three sprint trials with four to five minutes of recovery time between trials at 09:00 (morning session), 17:00 (evening session) and 21:00 (night session). The procedure was repeated the following day. A warm-up was applied prior to each session. The best performance of the three trials based on the RT was selected as the best neuromuscular function of the participant and used for further analysis.

\subsection{Reaction Time and Spatiotemporal Variables}

The sprint start began with a signal given by a starting device (ReacTime- Lynx System Developers, Inc). The ReacTime unit consists of a block sensor mounted with a monitor to a starting block and equipped with a loudspeaker to give the starting signal and record the reaction times. It was connected to one photocell gate at $3 \mathrm{~m}$ (Polifemo Radio LightMicrogate, Italy) that was triggered by the gun signal of the ReacTime device [14]. An optical measurement system (Microgate, OptoJump) [18] was also connected and synchronized with the ReacTime unit to assess spatiotemporal parameters such as the flight time and the length of the first step. The velocity of the first step was defined as the distance of the first step divided by the time elapsed. The first step was automatically calculated using the optical measure system by giving it the initial distance of the bases from the starting line, which was also the point where the optic bars were placed. Thus, the first step included the total distance from the base until the first ground contact between the optic bars.

\subsection{Premotor Time}

The gun of the starting device was wired to a circuit board with a voltage regulator that was used to convert the voltage of the $12-\mathrm{v}$ gun to $4.8 \mathrm{v} ; 5$ volts was the highest voltage that the EMG device could record. One of the EMG electrodes was modified to be easily connected and disconnected via an audio connector on the circuit board when the other side was plugged into the receptacle channels on the interface unit of the EMG wireless device (DELSYS EMG system, Myomonitor IV Wireless Transmission). After the gun signal, the gun cable was disconnected automatically from the circuit board to let the participant move freely. The electric (sound) pulse from the gun was recorded by the EMG device and used as the starting point in the electromyographic analysis (EMGworks 3.7 Delsys Inc.). Rectangular-shaped (19.8 mm X $35 \mathrm{~mm})$ bipolar surface electrodes (1-cm Ag conductors with an interelectrode distance of $1 \mathrm{~cm}$ ) were used to acquire the EMG activity from the rear and front legs and the gastrocnemius lateralis (GA), bicep femoris (BF) and rectus femoris (RF) muscles. Prior to the warm-up, the electrodes were placed on clean shaven skin overlying the muscles on the midpoint of the contracted muscle, which was based on the SENIAM recommendations for surface EMG electrode placement (www.seniam.org). An EMG data recorder at $1024 \mathrm{~Hz}$ was used to record the data. The reference electrode was placed over the iliac crest. The PMT was determined visually from unfiltered and rectified raw EMG data [19].

\subsection{Statistical Analysis}

Any systematic differences in the RT, PMT, 3-m time, first-step flight time, first-step length and first-step velocity across sessions were determined using a repeated measures analysis of variance (ANOVA), and the effect sizes were calculated as partial eta-squared $\left(\eta_{\mathrm{p}}{ }^{2}\right)$ [20]. To assess the relative and absolute reliability, the intraclass correlation coefficient (ICC) two-way mixed model of absolute agreement for the computation of ICC was used. The withinday reliability was determined from the sessions of the first test day (morning, evening, night). The between-day repeatability was calculated using the three sessions from the first test day and the three sessions from the second test day. The following ICC classifications were used: an ICC of 1.00 indicates perfect agreement and minimal variation, and an ICC $<0.67$ indicates a lack of absolute agreement and higher reliability [21]. The coefficient of variation (CV\%) was used to determine the within-participant variation [22]. A CV less than $10 \%$ and an ICC $>0.67$ was defined as a low variability. An ICC $<0.67$ or a $\mathrm{CV}>10 \%$ was interpreted as a moderate variability, and an $\mathrm{ICC}<0.67$ and a $\mathrm{CV}>10 \%$ was defined as a high variability [23]. All data are expressed as the means \pm SDs, and the significance level was set at $\mathrm{P}<0.05$.

\section{Results}

The purpose of the present study was to examine the reliability of sprint start variables both within a day and between days. There were statistically significant differences in the RT between the trials $\left(\mathrm{F}=3.46, \mathrm{p}=0.029, \eta_{\mathrm{p}}{ }^{2}=0.33\right)$, the PMT of the BF of the rear leg $(\mathrm{PMTrBF})(\mathrm{F}=4.79, \mathrm{p}=$ $\left.0.026, \eta_{\mathrm{p}}{ }^{2}=0.41\right)$ and the PMT of the RF of the front leg (PMTfRF) $\left(\mathrm{F}=5.31, \mathrm{p}=0.014, \eta_{\mathrm{p}}{ }^{2}=0.43\right)$. However, the results showed that there were nonsystematic differences in the PMT of the RF (PMTrRF) and the GA of the rear leg (PMTrGA), the BF (PMTfBF) and GA (PMTfGA) of the front leg, 3-m time, first-step flight time, first-step length and first-step velocity. The ICC scores of the within-day and between-day RT and PMTs ranged from 0.232 to 0.908 with moderate-to- high variability $(8.3 \%-21.5 \%)$. Both the within-day and between-day spatiotemporal markers showed higher reliability with lower variability from the reactive function markers. The within-day ICC scores ranged from 0.720 to 0.906 with low variability $(4.9 \%-8.3 \%)$ (Table 1$)$, and the between-day ICC scores ranged from 0.150 to 0.917 with low-to-moderate variability $(4.4 \%-9 \%)$. 
Table 1. Descriptive and reliability statistics of the within-day trials.

\begin{tabular}{|c|c|c|c|c|c|c|c|}
\hline & Mean \pm SD & $\min -\max$ & Cronbach's alpha & ICC single & ICC average & CV\% & Variability \\
\hline RT (s) & $0.167 \pm 0.022$ & $0.130-0.197$ & 0.836 & 0.503 & 0.752 & 13 & high \\
\hline \multicolumn{8}{|l|}{ Rear Leg } \\
\hline PMTrRF (s) & $0.281 \pm 0.032$ & $0.242-0337$ & 0.895 & 0.712 & 0.885 & 11.3 & moderate \\
\hline PMTrBF (s) & $0.143 \pm 0.031$ & $0.109-0.205$ & 0.947 & 0.687 & 0.868 & 21.5 & moderate \\
\hline PMTrGA (s) & $0.146 \pm 0.025$ & $0.112-0.187$ & 0.756 & 0.507 & 0.755 & 17.5 & high \\
\hline \multicolumn{8}{|l|}{ Front Leg } \\
\hline PMTfRF (s) & $0.310 \pm 0.026$ & $0.279-0.356$ & 0.789 & 0.298 & 0.580 & 8.3 & moderate \\
\hline $\operatorname{PMTfBF}(\mathrm{s})$ & $0.128 \pm 0.022$ & $0.106-0.165$ & 0.798 & 0.565 & 0.790 & 17.3 & high \\
\hline PMTfGA (s) & $0.152 \pm 0.03$ & $0.118-0.209$ & 0.745 & 0.474 & 0.730 & 19.6 & high \\
\hline \multicolumn{8}{|l|}{ 1st Step } \\
\hline Length (m) & $1.26 \pm 0.1$ & $1.06-1.40$ & 0.962 & 0.906 & 0.967 & 8.3 & low \\
\hline F-Time (s) & $0.568 \pm 0.028$ & $0.533-0.615$ & 0.887 & 0.744 & 0.897 & 4.9 & low \\
\hline Velocity (m/s) & $2.22 \pm 0.15$ & $1.94-2.41$ & 0.880 & 0.720 & 0.885 & 6.6 & low \\
\hline $3 \mathrm{M}(\mathrm{s})$ & $1.12 \pm 0.08$ & $1.00-1.24$ & 0.931 & 0.820 & 0.832 & 6.9 & low \\
\hline
\end{tabular}

Notes: ICC, intraclass correlation coefficient; CV, coefficient of variation; RT, reaction time; PMT, premotor time; RF, rectus femoris; BF, bicep femoris; GA, gastrocnemius lateralis; F-Time, flight time; 3M, 3 meters.

Between the morning trials, the ICC scores of the RT and PMTs varied from 0.232 to 0.766 with moderate-to-high variability. In contrast, the ICC scores of the spatiotemporal variables ranged from 0.150 to 0.853 with low-to-moderate variability (Table 2).

Table 2. Between-days morning-trials, descriptive and reliability statistics.

\begin{tabular}{llllllll}
\hline & Mean \pm SD & min - max & Cronbach's alpha & ICC single & ICC average & CV\% & Variability \\
\hline RT (s) & $0.173 \pm 0.021$ & $0.139-0.214$ & 0.969 & 0.766 & 0.867 & 12.3 & moderate \\
Rear Leg & & & & & & \\
PMTrRF (s) & $0.280 \pm 0.031$ & $0.218-0.337$ & 0.919 & 0.866 & 0.928 & 14.7 & moderate \\
PMTrBF (s) & $0.148 \pm 0.028$ & $0.104-0.177$ & 0.818 & 0.694 & 0.819 & 18.3 & moderate \\
PMTrGA(s) & $0.148 \pm 0.023$ & $0.104-0.184$ & 0.548 & 0.410 & 0.581 & 18.8 & high \\
Front Leg & & & & & & \\
PMTfRF (s) & $0.321 \pm 0.034$ & $0.251-0.334$ & 0.672 & 0.459 & 0.629 & 10.0 & high \\
PMTBF (s) & $0.129 \pm 0.019$ & $0.106-0.105$ & 0.791 & 0.684 & 0.812 & 14.2 & moderate \\
PMTfGA (s) & $0.155 \pm 0.033$ & $0.112-0.191$ & 0.457 & 0.314 & 0.478 & 19.7 & high \\
1st Step & & & & & & \\
Length (m) & $1.26 \pm 0.11$ & $1.07-1.37$ & 0.951 & 0.917 & 0.957 & 8.0 & low \\
F-Time (s) & $0.576 \pm 0.035$ & $0.517-0.614$ & 0.653 & 0.503 & 0.669 & 5.2 & moderate \\
Velocity (m/s) & $2.23 \pm 0.19$ & $1.94-2.14$ & 0.655 & 0.515 & 0.680 & 7.1 & moderate \\
3M (s) & $1.12 \pm 0.08$ & $0.99-1.21$ & 0.876 & 0.801 & 0.890 & 6.6 & small \\
\hline
\end{tabular}

Notes: ICC, intraclass correlation coefficient; CV, coefficient of variation; RT, reaction time; PMT, premotor time; RF, rectus femoris; BF, bicep femoris; GA, gastrocnemius lateralis; F-Time, flight time; 3M, 3 meters.

Between the evening trials, the ICC scores of the RT and PMT varied from 0.410- 0.866 with moderate-to-high variability. The ICC scores of the $3-\mathrm{m}$ time and first-step length were 0.801 and 0.917 , respectively, with low variability. Moderate variability was indicated for the first-step time and first-step velocity, and the ICC scores were 0.503 and 0.515 , respectively (Table 3).

Table 3. Between-days evening-trials, descriptive and reliability statistics.

\begin{tabular}{|c|c|c|c|c|c|c|c|}
\hline & Mean \pm SD & $\min -\max$ & Cronbach's alpha & ICC single & ICC average & CV\% & Variability \\
\hline RT (s) & $0.158 \pm 0.024$ & $0.112-0.183$ & 0.733 & 0.611 & 0.758 & 15.2 & high \\
\hline \multicolumn{8}{|l|}{ Rear Leg } \\
\hline PMTrRF (s) & $0.280 \pm 0.031$ & $0.239-0320$ & 0.449 & 0.267 & 0.422 & 11.3 & high \\
\hline PMTrBF (s) & $0.148 \pm 0.028$ & $0.107-0.193$ & 0.472 & 0.232 & 0.376 & 19.3 & high \\
\hline PMTrGA (s) & $0.148 \pm 0.023$ & $0.127-0.184$ & 0.901 & 0.756 & 0.86 & 15.6 & moderate \\
\hline \multicolumn{8}{|l|}{ Front Leg } \\
\hline PMTfRF (s) & $0.321 \pm 0.034$ & $0.276-0.369$ & 0.789 & 0.451 & 0.622 & 10.7 & high \\
\hline PMTfBF (s) & $0.129 \pm 0.019$ & $0.112-0.171$ & 0.909 & 0.759 & 0.863 & 14.8 & moderate \\
\hline PMTfGA (s) & $0.155 \pm 0.033$ & $0.118-0.212$ & 0.523 & 0.337 & 0.504 & 21.1 & high \\
\hline \multicolumn{8}{|l|}{ 1st Step } \\
\hline Length (m) & $1.26 \pm 0.11$ & $1.05-1.42$ & 0.929 & 0.876 & 0.934 & 9.0 & low \\
\hline F-Time (s) & $0.576 \pm 0.035$ & $0.528-0.617$ & 0.250 & 0.150 & 0.261 & 6.1 & moderate \\
\hline Velocity $(\mathrm{m} / \mathrm{s})$ & $2.23 \pm 0.19$ & $1.83-2.50$ & 0.932 & 0.867 & 0.927 & 8.6 & low \\
\hline $3 \mathrm{M}(\mathrm{s})$ & $1.12 \pm 0.08$ & $0.99-1.23$ & 0.935 & 0.853 & 0.921 & 6.8 & low \\
\hline
\end{tabular}

Notes: ICC, intraclass correlation coefficient; CV, coefficient of variation; RT, reaction time; PMT, premotor time; RF, rectus femoris; BF, bicep femoris; GA, gastrocnemius lateralis; F-Time, flight time; 3M, 3 meters. 
Between the night trials, the ICC scores of the RT and PMTfGA were 0.612 and 0.638 , respectively, with high variability, and the PMT of the rest of the muscles showed moderate variability with ICC scores from 0.542 to 0.908 . The ICC score of the-first step length was 0.712 with low variability. Moderate variability was indicated for the 3-m time, first-step time and first-step velocity, and the ICC varied from 0.538 and 0.631 (Table 4).

Table 4. Descriptive and reliability statistics of the between-day/night trials.

\begin{tabular}{|c|c|c|c|c|c|c|c|}
\hline & Mean \pm SD & $\min -\max$ & Cronbach's alpha & ICC single & ICC average & CV\% & Variability \\
\hline RT (s) & $0.161 \pm 0.025$ & $0.139-0.214$ & 0.740 & 0.612 & 0.759 & 15.5 & high \\
\hline \multicolumn{8}{|l|}{ Rear Leg } \\
\hline PMTrRF (s) & $0.275 \pm 0.031$ & $0.235-0.333$ & 0.921 & 0.822 & 0.902 & 11.3 & moderate \\
\hline PMTrBF (s) & $0.129 \pm 0.031$ & $0.101-0.200$ & 0.93 & 0.86 & 0.925 & 23.8 & moderate \\
\hline PMTrGA (s) & $0.140 \pm 0.026$ & $0.104-0.183$ & 0.899 & 0.835 & 0.910 & 18.6 & moderate \\
\hline \multicolumn{8}{|l|}{ Front Leg } \\
\hline PMTfRF (s) & $0.293 \pm 0.024$ & $0.271-0.340$ & 0.686 & 0.542 & 0.703 & 8.0 & moderate \\
\hline PMTfBF (s) & $0.123 \pm 0.024$ & $0.103-0.164$ & 0.948 & 0.908 & 0.952 & 19.9 & moderate \\
\hline PMTfGA (s) & $0.149 \pm 0.024$ & $0.120-0.190$ & 0.758 & 0.638 & 0.779 & 15.9 & high \\
\hline \multicolumn{8}{|l|}{ 1st Step } \\
\hline Length (m) & $1.25 \pm 0.11$ & $1.05-1.37$ & 0.816 & 0.712 & 0.832 & 8.2 & low \\
\hline F-Time (s) & $0.566 \pm 0.025$ & $0.532-0.606$ & 0.672 & 0.538 & 0.700 & 4.4 & moderate \\
\hline Velocity (m/s) & $2.23 \pm 0.16$ & $1.93-2.46$ & 0.750 & 0.609 & 0.757 & 7.2 & moderate \\
\hline $3 \mathrm{M}(\mathrm{s})$ & $1.12 \pm 0.08$ & $1.02-1.26$ & 0.770 & 0.631 & 0.774 & 7.2 & moderate \\
\hline
\end{tabular}

Notes: ICC, intraclass correlation coefficient; CV, coefficient of variation; RT, reaction time; PMT, premotor time; RF, rectus femoris; BF, bicep femoris; GA, gastrocnemius lateralis; F-Time, flight time; $3 \mathrm{M}, 3$ meters.

\section{Discussion}

The time of day is considered to influence physiological and neurological functions of exercise performance via circadian rhythms. Chronobiological studies suggest that short-term performance and psychomotor performance are improved during the early afternoon when core temperature is at its highest level [24]. The primary aim of the present study was to evaluate the within-day and between-day reliability of a sprint start isolated from the running distance performance. The reactive process variables (PMT, RT) and the spatiotemporal variables (3-m time, first-step flight time, first-step length and first-step velocity) were used as markers of the sprint start performance. It was hypothesized that the within-day reliability would be higher than the between-day reliability and that the $3-\mathrm{m}$ time, first-step flight time, firststep length and first-step velocity could be more variable than the RT and PMT. The spatiotemporal markers showed a high degree of reliability with low within-day variability, a good-to-high degree of reliability with low-to-moderate between-day variability, and no evidence of any systematic bias observed among sessions. Our results prove the hypothesis that the within-day reliability of the spatiotemporal markers was slightly higher than the betweenday reliability. Recently, Standing and Maulder (2017) investigated the reliability of sprint acceleration via kinematic analysis. They indicated that kinematic variables might not have an effective role in sprint performance, and there was lack of reliability between the sessions of sprinting times and the flight time of the first three steps. In opposition to the previous study of Standing and Maulder (2017), our results indicated an acceptable within-day and between-day reliability for the 3-m time and first-step flight time. This may occur because of the extended familiarization sessions that were performed prior to the experiment.
Reasonable reliability was observed among reactive markers and confirms our second hypothesis. Most cases reported a lack of absolute agreement and higher within-day as well as between-day reliability. The RT and PMT are more changeable markers than the spatiotemporal markers. Reactive functional markers could be influenced by many individual factors such as the arousal-attention state, practicefatigue effects, and motivational influences, which cannot be easily controlled during an experimental study. Moreover, the PMT may have been affected by surface inter/electrode displacement. Finally, it is unclear from the literature review whether the PMT is satisfactory concerning the total RT and final performance.

Recently, Paradisis (2013) revealed an association between the RT and 60-m final time in the indoor world games but not in the 100-m final time (outdoor), while Tønnessen et al. (2013) analyzed the IAAF's data of the world championship and reported that RT ability was related to the final running distance time $(100 \mathrm{~m})$. Finally, it is difficult to correlate the sprint start performance with the final time of running distance without taking into consideration the race tactics, the personal race record and the best reaction time.

\section{Conclusion}

The results of the present study lead us to conclude that well familiarized male participants can perform a reliable sprint start performance both within a day and between days even though reactive markers have a high within-day and betweenday variability. Further research is required to determine the dynamic role of the above markers at the block starting action. It is recommended that sports scientists and trainers should familiarize the participants/athletes prior to a research study or an athletic event, and they can use spatiotemporal markers as a valid method of sprint start assessment. 


\section{References}

[1] M. J. Harland and J. R. Steele, 'Biomechanics of the sprint start', Sport Med, vol. 23, no. 1, pp. 11-20, 1997.

[2] A. Mero and P. V. Komi, 'Reaction time and electromyographic activity during a sprint start', Eur J Appl Physiol Occup Physiol, vol. 61, no. 1-2, pp. 73-80, 1990.

[3] J. Slawinski et al., 'Segment-interaction in sprint start: Analysis of 3D angular velocity and kinetic energy in elite sprinters', J Biomech, vol. 43, no. 8, pp. 1494-1502, 2010.

[4] P. K. Schot and K. M. Knutzen, 'A Biomechanical Analysis of Four Sprint Start Positions’, Res Q Exerc Sport, vol. 63, no. 2, pp. 137-147, Jun. 1992.

[5] C. Milanese, M. Bertucco, and C. Zancanaro, 'THE EFFECTS OF THREE DIFFERENT REAR KNEE ANGLES ON KINEMATICS IN THE SPRINT START', Biol Sport, vol. 31, no. 3, pp. 209-215, Jul. 2014.

[6] M. T. G. Pain and A. Hibbs, 'Sprint starts and the minimum auditory reaction time', $J$ Sports $S c i$, vol. 25, no. 1, pp. 79-86, 2007.

[7] J. Botwinick and L. W. Thompson, 'Premotor and motor components of reaction time.', J Exp Psychol, vol. 71, no. 1, pp. 9-15, Jan. 1966.

[8] L. Nuri, A. Shadmehr, N. Ghotbi, and B. Attarbashi Moghadam, 'Reaction time and anticipatory skill of athletes in open and closed skill-dominated sport', Eur J Sport Sci, vol. 13, no. 5, pp. 431-436, 2013.

[9] G. Markovic, I. Jukic, D. Milanovic, and D. Metikos, 'Effects of Sprint and Plyometric Training on Muscle Function and Athletic Performance', J Strength Cond Res, vol. 21, no. 2, p. 543, 2007.

[10] E. Tønnessen, T. Haugen, and S. A. I. Shalfawi, 'Reaction time aspects of elite sprinters in athletic world championships.', J Strength Cond Res, vol. 27, no. 4, pp. 88592, Apr. 2013.

[11] E. J. Bradshaw, P. S. Maulder, and J. W. L. Keogh, 'Biological movement variability during the sprint start: Performance enhancement or hindrance?', Sport Biomech, vol. 6, no. 3, pp. 246-260, 2007.

[12] M. Coh and K. Tomazin, 'Kinematic analysis of the sprint start and acceleration from the blocks', New Stud Athl, vol. 21, no. 3, pp. 23-33, 2006.
[13] M. Otsuka, T. Kurihara, and T. Isaka, 'Effect of a wide stance on block start performance in sprint running', PLoS One, vol. 10 , no. 11,2015

[14] A. Smirniotou, C. Katsikas, G. Paradisis, P. Argeitaki, E. Zacharogiannis, and S. Tziortzis, 'Strength-power parameters as predictors of sprinting performance', J Sports Med Phys Fitness, vol. 48, no. 4, pp. 447-454, 2008.

[15] M. Jahanshahi, 'Chapter 15 Reaction time as an index of motor preparation/programming and speed of response initiation', 2003, pp. 203-229.

[16] N. Guissard, J. Duchateau, and K. Hainaut, 'EMG and mechanical changes during sprint starts at different front block obliquities', Medicine \& Science in Sports \& Exercise, vol. 24, no. 11. pp. 1257-1263, 1992.

[17] I. Kesoglou et al., 'Acute Effects of Specific Actions after the “ On Your Marks " Command', Biol Exerc, vol. 12, no. 1, pp. 55-68, 2016.

[18] J. F. Glatthorn, S. Gouge, S. Nussbaumer, S. Stauffacher, F. M. Impellizzeri, and N. A. Maffiuletti, 'Validity and reliability of optojump photoelectric cells for estimating vertical jump height', J Strength Cond Res, vol. 25, no. 2, pp. 556-560, 2011.

[19] R. Sokolova, O. Rukšènas, and B. Burle, 'RESPONSERELATED FACTORS IN REACTION TIMETO STIMULUS ONSET AND OFFSET TASKS', Psychology, vol. 48, pp. 79$89,2013$.

[20] S. Nakagawa, "A farewell to Bonferroni: the problems of low statistical power and publication bias', Behav Ecol, vol. 15, no. 6, pp. 1044-1045, 2004.

[21] K. O. Mcgraw and S. P. Wong, 'Forming Inferences About Some Intraclass Correlation Coefficients Forming Inferences About Some Intraclass Correlation Coefficients', Psycological Methods, vol. 1, no. 1, pp. 30-46, 1996.

[22] W. G. Hopkins, 'Measures of Reliability in Sports Medicine and Science', Sport Med, vol. 30, no. 1, pp. 1-15, 2000.

[23] E. Bradshaw, P. Hume, M. Calton, and B. Aisbett, 'Reliability and variability of day-to-day vault training measures in artistic gymnastics.', Sports Biomech, vol. 9, no. June, pp. 79-97, 2010 .

[24] B. Drust, J. Waterhouse, G. Atkinson, B. Edwards, and T. Reilly, 'Circadian Rhythms in Sports Performance-an Update', Chronobiol Int, vol. 22, no. 1, pp. 21-44, Jan. 2005.

[25] G. P. Paradisis, 'Reaction Time and Performance in the Short Sprints', Nsa, vol. 28, no. 1/2, pp. 95-103, 2013. 